16 | 2013

NOVECENTO... E DINTORNI

« On ne naît pas... on le devient "

\title{
Closet, ma con vista. I queer studies e l'Italianistica
}

Closet... with a View. Queer and Italian Studies

\section{Eleonora Pinzuti}

\section{OpenEdition}

\section{Journals}

Edizione digitale

URL: http://journals.openedition.org/cei/1081

DOI: 10.4000/cei.1081

ISSN: 2260-779X

Editore

UGA Éditions/Université Grenoble Alpes

\section{Edizione cartacea}

Data di pubblicazione: 30 juin 2013

Paginazione: 51-64

ISBN: 978-2-84310-245-5

ISSN: $1770-9571$

Notizia bibliografica digitale

Eleonora Pinzuti, «Closet, ma con vista. I queer studies e l'Italianistica», Cahiers d'études italiennes [Online], 16 | 2013, online dal 15 décembre 2014, consultato il 26 mars 2021. URL: http:// journals.openedition.org/cei/1081 ; DOI: https://doi.org/10.4000/cei.1081 


\title{
CLOSET, MA CON VISTA. I QUEER STUDIES E L'ITALIANISTICA
}

\author{
Eleonora Pinzuti \\ Università di Firenze
}

Il linguaggio della sessualità non solo si interseca con, ma trasforma anche gli altri linguaggi e le relazioni tramite le quali noi conosciamo.

Eve Kosofsky Sedgwick, Epistemology of the Closet

\section{La letteratura del closet $^{\mathrm{I}}$ : cruising literature}

Non va fatta una distinzione binaria fra quel che si dice e quel che non si dice; bisognerebbe invece cercare di determinare le diverse maniere di non dire, come si distribuiscono quelli che possono e quelli che non possono parlarne, quale tipo di discorso è autorizzato o quale forma di discrezione è richiesta per gli uni e per gli altri. Non c'è uno, ma più tipi di silenzio, ed essi fanno parte integrante delle strategie che sottendono ed attraversano i discorsi. ${ }^{2}$

Michel Foucault, in questo brano della Volontà di sapere, concettualizza l'atto del silenzio come un evento discorsivo: in altri termini presuppone, all'interno delle strategie di silenziamento, una forza contenutistica le cui modalità operative vanno lette nel rovescio del dettato, nella coerenza strutturale fra il materiale espresso (la common opinion) e quello occultato. In questo senso, il taciuto, l'omesso, il punto di frattura della

I. Con questo termine si cita, indirettamente, un libro famosissimo di Eve Kosofsky Sedgwick, Epistemology of the Closet, New York, London, Harvester Wheatsheaf, 1991 (prima edizione 1990), che vede finalmente oggi la traduzione italiana (cfr. n. 52).

2. Michel Foucault, La volontà di sapere. Storia della sessualità I, trad. it. di Pasquale Pasquino e Giovanna Procacci, Milano, Feltrinelli, 2010 (edizione originale 1976), p. 28. 
comunicazione indicano il luogo stesso dell' intero messaggio, composto da ciò che dico ma soprattutto da quello che scelgo di non dire. Questa "teoria di inversione delle logiche» ${ }^{3}$ è dunque sì interessata alla negazione del soggetto perturbante, ma soprattutto alla produzione di strategie di contenimento che, definendo a priori la realtà simbolica, ne azzerano gli ulteriori eccessi di significazione. In tal senso il «ripostiglio» sedgwickiano (il closet) diventa il non-luogo di accumulo tematico e di addensamento semiologico di tutto ciò che non deve toccare il livello prospiciente del testo, ma che è vincolato alla giacenza negli interstizi dell'espressione. Ancor più, il ripostiglio diventa una co-funzione inerente al testo affinché questo possa prodursi nella sua capacità di comunicazione 'totale': quel che posso dire è infatti strettamente vincolato (e sorretto) da ciò che non posso dire. Anzi, è proprio quel che non posso dire che convalida quanto sto dicendo. Infatti, poiché il testo è «un mezzo di verifica intersoggettiva che fa appello alla scrittura testuale come medium sociale e come valore pubblico» ${ }^{4}$, la sua coerenza ideologica è sostenuta dai due elementi (dire - non dire) già previsti all'interno della comunicazione.

È peraltro noto come il soggetto marginalizzato, là dove non abbia avuto accesso all'atto di parola se non per negare il proprio desiderio affermandolo in tralice, abbia dovuto inscenare strategie di dizione indirette in grado di offrire una primitiva forma di comunicazione codicizzata e perciò ambivalente:

una persona che è esclusa dall'universale, eppure appartiene comunque ad esso, parla a partire dalla condizione divisa dell'essere allo stesso tempo autorizzata e non autorizzata (e questa è la fine della possibilità di delineare un ben definito «luogo di enunciazione»). Quel parlare non è solo una semplice assimilazione a una norma esistente, perché quella norma è fondata sull'esclusione di colui o colei che parla e le cui parole mettono in questione la fondazione dell'universale stesso. Parlare e far vedere l'alterità all'interno della norma (l'alterità senza la quale la norma non "conoscerebbe se stessa») mostrano il fallimento della norma nel produrre l'ambito universale che essa permette e mostrano altresì ciò che potremmo mettere in rilievo come ambivalenza promettente della norma. ${ }^{5}$

3. Marco Pustianaz, Teoria gay e lesbica, in Teoria della letteratura. Prospettive dagli Stati Uniti, a cura di Donatella Izzo, Roma, Nuova Italia Scientifica, I996, pp. I09-I29; I2I.

4. Romano Luperini, Il dialogo e il conflitto. Per un'ermeneutica materialistica, Roma, Bari, Laterza, I999, p. I7.

5. Judith Butler, Parole che provocano. Per una politica del performativo, trad. it. di Sergia Adamo, Milano, Raffaello Cortina Editore, 2010 (edizione originale 1997), p. I30. 
Per la letteratura omosessuale ${ }^{6}$ — che d'ora in avanti renderò graficamente con (omo)sessuale, per sottrarre il termine al medicalismo ottocentesco e alla fissazione del dualismo - la produzione di un sottocodice comunicativo è stata a lungo unica enunciazione possibile. Infatti quando emerge sulla pagina il personaggio omosessuale ${ }^{7}$ si attivano due retoriche, opposte e co-producenti: quella prodotta dal discorso eterosessuale, che leggeva il gay o la lesbica come s/oggetti medicalizzati, e quella autoprodotta, che, mischiando le strategie informative, tentava di proporre, usando spesso forme sotto-comunicative, la propria versione esperien$z_{i a l e}^{8}$. Non va dimenticato che proprio fra la fine dell'Ottocento e i primi del Novecento, si cristallizzarono due retoriche: la prima prodotta dalla visione medica ${ }^{9}$, che dava dell'omosessuale una descrizione nosologica, la seconda scaturita dall'esperienza stessa dell'(omo)sessualità.

La visione medica come forma di 'sapere' può essere letta come una sorta di teoria della in-formazione che genera la formazione del soggetto grazie alla replicazione delle modalità culturali motrici delle credenze e delle azioni nel mondo. Se infatti «il messaggio linguistico [...] proviene sempre dall'Altro» ${ }^{\mathrm{IO}}$, la costruzione dell'as/soggettamento ${ }^{\mathrm{II}}$ si modula figurandosi e con-figurandosi all'interno dei perimetri che la conoscenza eterodiretta produce.

Intorno alle (omo)sessualità e alle loro pratiche di desiderio, la colonizzazione del collettivo dominante si è mossa all'interno dei paradigmi propri dell''alterità genericamente intesa: il monstruosum omosessuale è stato descritto in virtù di 'conoscenze' pregiudiziali che andavano a

6. Intendo con il sintagma tutta quella produzione testuale che rivela un alto gradiente tematico attorno alla cosiddetta 'omosessualità. È piuttosto frequente l'affermazione che vede nella declinazione aggettivale una forma di 'ghettizzazione'. Ma se la specificazione è ritenuta in sé ghettizzante allora anche le diciture «letteratura italiana» o «letteratura vittoriana» dovrebbero risentire di una forma di 'ghettizzazione', che invece, ovviamente, non subiscono. In realtà la presenza aggettivale evidenzia esclusivamente un elemento narratologico di somiglianza-appartenenza che designa il 'tema', sia esso linguistico o narratologico (penso anche al «romanzo poliziesco", per portare un esempio). Ma, mentre su altre categorizzazioni teoriche non si percepisce nessuno 'svilimento', per la letteratura a tematica omosessuale la specificazione è letta come segno deteriore, evidenziando così l'introiezione di un disvalore insito nel termine "omosessuale».

7. Cfr. Michel Foucault, La volontà di sapere. Storia della sessualità I, cit., p. 42.

8. Per questo sia lecito il rimando a un mio saggio che analizza la questione: Eleonora Pinzuti, Queering Proust. Rhetorical Incoherences, Performance and Gender In-Version in In Search of Lost Time, in Queer Crossings, Theories, Bodies, Texts, a cura di Silvia Antosa, Milano, Udine, Mimesis, 2012, pp. I39-I59.

9. Cfr. Graham Robb, Sconosciuti. L'amore e la cultura omosessuale nell'Ottocento, trad. it. di Maria Baiocchi, Roma, Carocci, 2003 (edizione originale 2003), in part. pp. 49-72.

Io. Jacques Lacan, Della struttura come immissione di un'alterità, presupposto di qualsiasi oggetto, in La controversia sullo strutturalismo, a cura di Richard Macksey ed Eugenio Donato, Napoli, Liguori, I975 (edizione originale 1970), pp. 269-28I; 269.

II. Cfr. Judith Butler, La vita psichica del potere. Teorie della soggettivazione e dell'assoggettamento, a cura di Carla Weber, trad. it. di Elena Bonini e Carlotta Scaramuzzi, Roma, Meltemi, 2005 (edizione originale 1997). 
formare il 'sapere scientifico' da un lato e quello 'retorico' dall'altro, rendendo il soggetto oggetto delle funzioni proiettive del perturbante.

In tal senso il testo diviene matrice di riferimento e strumento atto a educare la modellizzazione del soggetto/personaggio, il tessuto connettivo sul quale si innestano le capacità di raffigurazione sociale del soggetto minoritario. Ma questo primitivo modello metaforico ha prodotto anche la propria auto-narrazione, instaurando quella «relazione del ripostiglio", cioè il luogo di accumulo di materiali in-dicibili, che ha scontato tre principali modalità di inter-dizione: la censura, l'autocensura, il mascheramento.

Di questa triade, la censura è la più innocua. Perdendo in anticipo la funzione di medusizzazione, di blocco/azzeramento del significante, e rivelando la propria strategia economico-simbolica, la censura espone il proprio dispositivo: lo offre e lo mostra nella sua natura performativa. Di contro, l'autocensura, agendo invece come 'soggettività collettiva' atta ad auto-regolamentare l'espressione prima ancora che questa raggiunga il proprio referente, offre al teorico l'istanza della regola ${ }^{\mathrm{I}}$. Per quel che riguarda la letteratura a tematica (omo)sessuale, basti ricordare qui Forster e il suo Maurice, non a caso dedicato a un "Happier year»" ${ }^{13}$, cioè quando ci sarebbero state "quelle "condizioni di possibilità" di un determinato fenomeno registrato nel corso della lettura o del commento» ${ }^{14}$ atte a esplicitare e accogliere il messaggio del romanzo. Ma si pensi, in ambito italiano, a Saba e alla «impubblicabilità» ${ }^{15}$ del «mondo meraviglioso» ${ }^{16}$ di Ernesto che, scritto tra il maggio e il giugno del 1953 in una "crisi di maternità» ${ }^{17}$, vedrà la luce della stampa solo nel 1975 a cura della figlia. $\mathrm{O}$ si rifletta sulla variantistica palazzeschiana nel Codice di Perelà, dove vengono espunti, dall'edizione del $\mathrm{I}^{\mathrm{II}} \mathrm{I}^{\mathrm{I}}$, i riferimenti espliciti all'omosessualità (pederasta; contro natura; terzo sesso) che non compariranno nell'edizione del $1958^{19}$.

I2. Michel Foucault, La volontà di sapere. Storia della sessualità I, cit., p. 74.

13. Cfr. Edward Morgan Forster, Maurice, a cura di Luisa Bulgheroni, trad. it. di Marcella Bonsanti, Milano, Garzanti, I987 (edizione originale I97I).

I4. Romano Luperini, Il dialogo e il conflitto. Per un'ermeneutica materialistica, cit., p. 25.

15. "Mio caro Pierantonio, se ti ho detto che il romanzo è impubblicabile, vuol dire che lo è. Su questo punto sono d'accordo anche le due o tre persone che ne hanno letti (ascoltato leggere) alcuni brani.» (Umberto Saba, A Pier Antonio Quarantotti Gambini, 25 agosto 1953, in La spada d'amore. Lettere scelte 1902-1957, a cura di Aldo Marco Vecchio, presentazione di Giovanni Giudici, Milano, Mondadori, 1983, p. 260.)

I6. Cito dall'esergo in Umberto Saba, Ernesto, a cura di Maria Antonietta Grignani, Torino, Einaudi, I995, p. 3 .

17. Maria Antonietta Grignani, Introduzione, in Umberto Saba, Ernesto, cit., pp. v-xxiv; v.

I8. Aldo Palazzeschi, Il codice di Perelà, Milano, Edizioni futuriste di «Poesia», I9II, p. 250.

19. Cfr. Aldo Palazzeschi, Il codice di Perelà (1958), in Tutti i romanzi, vol. I, a cura e con introduzione di Gino Tellini e un saggio di Luigi Baldacci, Milano, Mondadori, 2004, pp. II87-II9I. 
È dunque nel gioco dell'indizio, nella mascherata, che il discorso omosessuale ha prodotto, almeno fino al dopoguerra, le sue funzioni di significato, perché è nell'open secret, nell'azione di due retoriche, volte una al lettore "co-giudicante» e l'altra al lettore "co-producente» ${ }^{20}$, che si è giocata la possibilità di esaudire quelle 'condizioni di possibilità' latenti nel testo:

Tu che ti occupi principalmente di letteratura... Diciamo di letteratura 'mascherata'! Perché mascherata? Perché cerco di studiare i meccanismi... meglio, gli schemi di confezione testuale concepiti per accogliere istanze gay e farle passare in epoche e ambienti che non erano disposti ad accoglierle. ${ }^{21}$

Non a caso infatti la letteratura omosessuale ha fatto uso dell'intertestualità come net in grado di sovra-significare l'appartenenza tematicoculturale, sviluppando al proprio interno quella necessità di cruising ben noto alla cultura gay ${ }^{22}$. L'amore che non poteva dire il suo nome ${ }^{23}$, che esisteva dunque in virtù esclusiva della sua negazione aperta, necessitava di uno sguardo attento a cogliere tra gli interstizi del testo, fra le sue nervature macro- e microstrutturali, in quelle pieghe retoriche che fanno di un romanzo un romanzo a tematica omosessuale (penso, solo per fare un esempio, a The Picture of Dorian Gray ${ }^{24}$ ). Ma se la letteratura è capace di sovra-significare comunque (per questo, appunto, le censure), la volontà tacitante della critica e dell'epistemologia è assai più efficiente (e dunque più pericolosa). Interpretare significa infatti non solo decidere quel che il

20. Hans Robert Jauss, La teoria della ricezione, in Teoria della ricezione, a cura di Robert Charles Holub, trad. it. di Costanzo Di Girolamo et al., Torino, Einaudi, 1989, pp. 3-26; I4.

2I. Franco Buffoni, Zamel, Milano, Marcos y Marcos, 2009, pp. I67-I68. Curiosamente in Zamel viene incipitariamente descritta una biblioteca, i cui titoli vengono citati in dettaglio: la volontà descrittiva è quella di dar conto della letteratura 'medica' intorno all'omosessualità. In un altro lavoro in prosa di Buffoni a gradiente autobiografico, Reperto 74 e altri racconti (Arezzo, Zona, 2008), l'autore cita un capitolo iniziale del raccontoreperto nel quale, dice Buffoni, «[...] avevo stipato tutte le mie conoscenze di cultura omosessuale [...] infarcito di citazioni mediche e filosofiche» (pp. 5-6), che sarà espunto nella edizione per la stampa. L'impressione è che quella 'conoscenza testimoniale' sia rifluita nelle prime pagine di Zamel, ma mi riprometto una indagine ulteriore.

22. Intendo utilizzare questo termine in senso teorico, lasciandogli tutto il suo significato desiderante, cioè di ricerca — oltre le apparenze — della disponibilità del testo a rispondermi. Il cruising è peraltro termine inglese che fa parte della cultura gay, che descrive «someone [who] looks for sexual partners in bars, restaurants, on the streets" (Longman Dictionary) ed è conosciuto in ambiente italiano con il termine battuage (cfr. Gay. La guida italiana in Iso voci, a cura di Daniele Del Pozzo e Luca Scarlini, prefazione di Gianni Vattimo, Milano, Mondadori, 2006).

23. Richiamo qui i noti versi scritti da Lord Alfred Douglas e pubblicati sul «Chameleon» nel dicembre del I894 (cfr. Oscar Wilde, Opere, a cura di Masolino D’Amico, Milano, Mondadori, 2006, p. I382 e n.). Alfred Douglas è il Bosie di Oscar Wilde, a cui è anche indirizzato il De Profundis, che incìita "Caro Bosie» (cfr. Oscar Wilde, De Profundis, in Opere, cit., p. 1393).

24. Si legga il giudizio dato sull'opera in Dossier Oscar Wilde, a cura di William Badford, traduzioni di Lorenzo Ruggiero, Bollate, Kaos edizioni, 20Io, pp. 23-24. 
testo dice, bensì decidere cosa far dire al testo. Oltre a questo livello 'consapevole' (nego che il testo possa dire ciò che ritengo immorale o contrario alla norma per lasciare al testo tutta la sua presunta innocenza/purezza di 'verbo' non incarnato: la metafisica della lettera), esiste un livello non consapevole di opacizzazione. Infatti, se non conosco gli elementi per decrittare il codice, per accedere oltre il velame ${ }^{25}$, sarò incapace di attualizzarne i significati possibili. È quindi nella critica alla volontà speculativa che si spezza il cortocircuito del closet. Bisogna dunque aguzzar «le ciglia/ come 'l vecchio sartor fa ne la cruna ${ }^{26}$ e osservare i movimenti dell'epistemologia.

\section{La critica del closet: cruising epistemology}

Non è l'esperienza che organizza l'espressione, ma, al contrario, è l'espressione che organizza l'esperienza, le dà una forma e ne definisce l'orientamento.

\section{Michail BaCHTIN, Linguaggio e scrittura}

Da noi arriva sempre tutto molto tardi e perché una innovazione tocchi l'istituzione accademica, che è quanto di più conservatore possa esserci, deve passare molto tempo, deve consolidarsi. Invece negli Stati Uniti, se si produce e si diffonde una tendenza critica, come appunto i gay and lesbian studies, la queer theory, i gender studies, subito trovano spazio nelle università. Non è un caso che le poche cose che si fanno da noi, le fanno gli americanisti. Già è un po' diverso per i gender studies, legati alla cultura femminista che è un po' più radicata in Italia ${ }^{27}$.

Così Massimo Fusillo in una intervista a Francesco Gnerre. Di fatto, nell'ambito della letteratura italiana si è assistito a due movimenti opposti e contrari: da un lato il discorso letterario che includeva nel suo racconto l'(omo)sessualità si è imposto di forza ai lettori, per qualità (Pasolini, Arbasino, Tondelli) e per accumulazione tematica (Penna, Bellezza, Testori) ${ }^{28}$, dall'altro l'istituzione accademica ha risposto silenziandone la ricaduta

25. Se il lemma è dantesco, presente in una famosa terzina dell'Inferno (IX, vv. 6I-63), Eco ha dedicato agli «adepti del velame» pagine interessanti. Cfr. Umberto Eco, I limiti dell'interpretazione, Milano, Bompiani, I990, pp. 89-95. Io lo uso qui in senso parodico.

26. I versi sono ovviamente di Dante, e descrivono il momento in cui Brunetto Latini riconosce il poeta, suo antico allievo (Inferno, XV, vv. 2O-2I). Per uno studio su Dante e l'omosessualità, cfr. Massimiliano Chiamenti, Dante sodomita?, in "L'Alighieri», 34, 2009, pp. I3I-I46.

27. L'altro e lo stesso, intervista di Francesco Gnerre a Massimo Fusillo: <www.pasolini.net/saggistica_Massimo Fusillo_altro-e-io-stesso.htm> (data di ultima consultazione 8 dicembre 20I2).

28. Si veda l'insuperato Francesco Gnerre, L'eroe negato. Omosessualità e letteratura nel Novecento italiano, Milano, Baldini \& Castoldi, 2000. 
teorica e interrogativa. Di fatto, opacandone l'esistenza o azzerando le implicazioni storiche ed epistemologiche a mera declinazione (spesso ideologicamente direzionata) all'interno del canone mainstream, l'accademia ha riprodotto il proprio neutro eterosessuale ${ }^{29}$, spacciato per universale, poiché «il confine che produce il dicibile escludendo certe forme di discorso diventa un'operazione di censura esercitata proprio dal postulare l'universale» $3^{30}$.

Quel che a lungo è mancato è stata in sostanza una 'sub-versione' della epistemology of the closet, una presa in carico delle significazioni (culturali, politiche, teoriche, storico-critiche) che i testi esprimevano e il riconoscimento di un contro-valore al discorso omosessuale.

Il ripostiglio epistemologico in cui finivano dunque i materiali relativi alla cultura (omo)sessuale ha continuato ad accumulare rimasugli 'fantasmatici', tratti residuali non ritenuti 'dicibili', né tantomeno utili a interpretazioni che imponevano canonizzazioni e 'beatificazioni' testuali. Le ragioni di un simile atteggiamento, in Italia ben più marcato che altrove, sono molteplici: da un lato è registrabile la diffidenza epistemologica dell'università italiana verso saperi interdisciplinari e indisciplinati, dall'altro può aver funzionato da barriera la produzione genericamente in inglese di questi studi (lingua meno frequentata dagli italianisti che per lo più si sono formati negli anni Cinquanta e Sessanta studiando più il francese che l'inglese); infatti, non a caso, l'anglistica è stato il settore che per primo ha tradotto queste ricerche in Italia.

Ma credo sia da riflettere anche intorno alla pruderie tutta italiana verso gli studi inerenti la sessualità prodotti in Francia e in America, assenti dai nostri corsi universitari, come assenti restano cattedre di Sexuality e Gender comuni fuori dall'Italia. Inoltre, la ricezione di un femminismo a tratti essenzialista e differenzialista non ha intaccato la costruzione duale $\mathrm{m} / \mathrm{f}$, lasciando inalterate quelle strutture di mantenimento delle stereotipie di genere che affliggono e ricadono sulla lettura qualunquista delle (omo)sessualità. Non ultimo, una eccellente tradizione ecdotica (la scuola degli Avalle, Segre, Contini e di Rosanna Bettarini, filologa d'eccellenza recentemente scomparsa, per intendersi, che resta insuperata) può aver agito da contrappeso per letture eccedenti, benché la filologia sia ancor oggi lo strumento più acuto nella decrittazione storico-culturale dei testi.

29. Uso questo sintagma richiamando in tralice il «neutro universale maschile» di tanta critica femminista. 30. Judith Butler, Parole che provocano. Per una politica del performativo, cit., p. I28. 
Questo insieme di circostanze e condizioni ha fatto sì che la cultura italiana e l'Italianistica in particolare rimanessero vincolate a paradigmi storicistici o a letture che espungevano la componente sessuale o di genere dalle interpretazioni critiche.

In questo senso si comprende meglio perché studiare le strategie narrative del 'romanzo lesbico' o l'intertestualità dei personaggi omosessuali sia parso fino a oggi compito di un militante e non di uno studioso (figurarsi di una studiosa). In effetti c'è stata, e temo ci sia ancora, una sorta di tassonomia inerente ad 'argomenti accademici' (= seri) e ad 'argomenti non accademici' (= non seri) tradizionalmente decisi. Ora, se per tradizione intendiamo i prodotti culturali e la dizione intorno ai loro oggetti espressivi, ci rendiamo conto che la loro stessa selezione «rivela a priori lo sguardo amministrativo che, dall'alto, raccoglie, suddivide, soppesa» ${ }^{3 \mathrm{I}}$, denunciando di fatto la posizione egemonica di chi decide cosa è importante studiare. Per il/la teorico/a che voleva attivare queste interrogazioni, andando così a colmare una enorme voragine, questo ha significato non solo la negazione del proprio simbolico e un ovvio depauperamento di processi di significazione dei testi, ma spesso ha implicato anche il forzato abbandono della ricerca da parte di giovani studiosi/e che non hanno trovato in Italia nessuno supporto:

[The question involves also] the political question of how the academic staff is recruited. This has virtually barred in the past any access to gays and lesbians openly engaged in gay research (though not to openly gay teachers). Visibly gay teachers are obviously there, not many of them maybe, but still there, only they are not even remotely interested in gay studies, in that they tend to see it as parochially 'gayaffirmative', a partiality that sounds alien to the presumed disinterestedness of 'culture' and to its apparent universality. But the fact that gay studies lack gay scholars is not just a result of the complicity of those gay academics who work with 'liberalhumanist' definitions of culture and are bound by traditional loyalties to their disciplines. It is also specifically the product of a system which in practice has stopped gay scholars (scholars with a commitment to gay studies) from ever getting in. ${ }^{32}$

Questa situazione è stata più cogente nell'Italianistica. Giulio Iacoli non a caso parla, nel suo bel saggio dedicato agli studi culturali e ai queer studies $^{33}$, di «ultima chiamata per l'Italianistica»:

3I. Theodor Adorno, Scritti sociologici, trad. it. di Anna Marietti Solmi, Torino, Einaudi, 1976, p. II5.

32. Marco Pustianaz, The White Hole of Gay Studies in Italy, <http://old.www.gay-web.de/fluss/konferenz/ pustianaz.html> (data di ultima consultazione 8 dicembre 20I2).

33. La resa non in corsivo del lemma "queer» e dei successivi sintagmi "queer studies» o "studi queer» è da intendersi una scelta teorica: come infatti ho avuto modo di affermare altrove, "potrebbe essere utile rinunciare al corsivo, che evidenzia ancora, tachigraficamente, una sua estraneità al discorso (almeno in Italia)» (Eleonora 
Simili atteggiamenti di chiusura, oltre a non condurre a cogliere come le basi dell'interpretazione letteraria vadano costantemente rinegoziate di fronte all'apporto di metodologie ulteriori, e virtualmente più idonee ad applicarsi a certe classi di testi, contribuiscono ad arenare nello status quo ante una critica avviluppata, con un gusto quasi masochistico, nella discussione dei suoi diuturni episodi di crisi. Non solo: ratificano implicitamente una condizione di oppressione simbolica ai danni di soggettività storicamente marginalizzate e represse, e dunque escluse dall'opportunità di esercitare pubblicamente giudizi critici, la quale va ad affiancarsi a un'oppressione materiale che nel nostro paese perdura in maniera drammatica. ${ }^{34}$

Pare del resto pacifico che, senza l'attivazione di indicazioni operative e processuali degli strumenti euristici oramai diffusissimi in Europa e in America, la critica nostrana non può che ripiegarsi in una agonica ripetizione di se stessa. Infatti una corretta lettura di soggettività e di sessualità, di embodiment sottratti alla forzata eterosessualizzazione, è in grado di produrre edizioni critiche corrette (mi riferisco, solo per fare un esempio, alle Rime di Michelangelo, ma penso anche a Benedetto Varchi), così come di rileggere criticamente i travestimenti di Palazzeschi o le strategie di occultamento di Gadda: quel che bisogna indagare sono, insomma, la somatic fiction e le sue materializzazioni testuali.

Lapplicazione del sapere queer è infatti necessaria non solo e non tanto per una pluridialogicità critica, quanto per una corretta lettura dei dati testuali e per una onesta visione argomentativa della storia della letteratura; ritenere inoltre gli studi queer produzione esclusiva di una subcultura depaupera non solo l'intero atto conoscitivo su e intorno ai testi, ma denuncia la posizione dell'accademia che troppo a lungo ha relegato a 'particolarizzazione' un sapere che deve essere diffuso e comunitario.

Visto che la funzione dialogica della letteratura si è sempre mossa nell'inter-locuzione, sono quanto mai necessarie una epistemologia e una comparatistica che producano interrogazioni impreviste e che rileggano le stesse funzioni teoriche a partire da posizioni supplementari. In questo senso, la lunga sottrazione dai manuali d'uso universitario dei queer studies non è un danno o una mancanza che va a detrimento delle identità left (che, di fatto, non esistono se non in riferimento a un sistema che le ritiene tali) quanto a nocumento di una intera area degli studi, impoverendo i testi nella loro intrinseca polisemia. A conferma di ciò basta dare un'occhiata ad alcuni dei manuali a uso universitario per accorgersi come

Pinzuti, Queer, in Queer in Italia. Differenze in movimento, a cura di Marco Pustianaz, Pisa, ETS, 20II, pp. II8-I22).

34. Giulio Iacoli, Carte dalla penombra. Studi letterari, studi culturali, studi queer, in «Moderna», 2, 2012, in corso di stampa. 
non siano affrontate, se non in senso meramente citazionale, le metodologie a cui mi riferisco.

Mentre negli Studi delle donne in Italia, a cura di Paola di Cori e Donatella Barazzetti, si trovano pagine a firma di Marco Pustianaz sugli Studi gay e lesbici35, così come va a Michele Cometa il merito di aver inserito nel suo importante Dizionario degli studi culturali pagine dedicate agli Studi gay e lesbici e agli Studi queer sempre a firma di Pustianaz ${ }^{36}$, Francesco Muzzioli nel suo Le teorie della critica letteraria dedica poche righe alla queer theory e soltanto all'interno delle critiche femministe: "Così un ramo si sviluppa negli studi dell'omosessualità (che hanno ricevuto negli Stati Uniti la sigla della "Queer Theory"), particolarmente indicati a recepire i temi del 'travestimento' e quindi le forme della parodia.» ${ }^{37}$ La latitanza è confermata anche in un altro volume, a uso sostanzialmente didattico, di Alberto Casadei, dal titolo La critica letteraria del Novecento, dove, nelle poche pagine dedicate alle novità di fine secolo, si legge

l'analisi della diversità sessuale ha infine generato ulteriori tendenze della critica, spesso etichettate come gender studies. Molteplici sono le diramazioni in questo settore, specie per l'attenzione riservata a vari tipi di scritture omosessuali: una raccolta di saggi come Opposite Sex, ben rappresenta questi sviluppi. ${ }^{38}$

Ancora, in un manuale specificatamente dedicato agli studenti, Teorie critiche del Novecento, i queer studies sono inseriti in un elenco di metodologie sensibilmente differenti:

L'ambito statunitense e anglosassone è sicuramente il contesto ideologicamente tra i più vivaci. Ma qui, in modo più marcato rispetto all'Italia e agli altri paesi, gli interessi femministi sono estesi anche alle questioni di individualità etniche, sessuali, razziali, e interculturali (perciò si parla di Cultural studies, Black studies, Queer studies, Lesbian studies). ${ }^{39}$

Che non si tratti di totale dis-conoscenza pare ovvio, né che i queer studies non siano pertinenti alla teoria della letteratura, come dimostrava meritoriamente Donatella Izzo che, in Teoria della letteratura. Prospettive

35. Marco Pustianaz, Studi gay e lesbici, in Gli studi delle donne in Italia, a cura di Paola di Cori e Donatella Barazzetti, Roma, Carocci, 200I, pp. 24I-257.

36. Cfr. Michele Cometa, Dizionario degli studi culturali, a cura di Roberta Coglitore e Federica Mazzara, Roma, Meltemi, 2004, pp. 435-440 e 44I-448.

37. Francesco Muzzioli, Le teorie della critica letteraria, Roma, Carocci, 2005 (prima edizione 1994), p. 22I.

38. Alberto Casadei, La critica letteraria del Novecento, Bologna, Il Mulino, 20oI, p. I63. Casadei utilizza il sintagma «diversità sessuale» senza virgolette, dando per implicite una normalità e una diversità.

39. Enza Biagini, Il poststrutturalismo, le teorie femministe e postfemministe. La critica genetica, in Teorie critiche del Novecento. Con antologia di testi, a cura di Enza Biagini, Augusta Brettoni, Paolo Orvieto, Roma, Carocci, 200I, pp. 253-315; 264. Nonostante una nuova edizione recentissima (Roma, Carocci, 20II), non sono state apportate modifiche o prodotti approfondimenti. 
dagli Stati Uniti, dedicava, nel lontano 1996, il già ricordato capitolo alla teoria gay e lesbica ${ }^{40}$.

Si tratta invece della predominanza di linee interpretative canoniche che a lungo non hanno lasciato spazio, né evidenziato alcun interesse, per altre declinazioni teoriche, inclinando verso un monologismo autoreferenziale. Se in un libro eccellente come Estetica della letteratura Massimo Fusillo dedica pagine informate sul camp/queer ${ }^{4 \mathrm{I}}$, nel recente Metodi e protagonisti della critica letteraria, informatissimo su altre metodologie, si legge: «un particolare sistema culturale è connesso sia all'identità omosessuale maschile, che è al centro dei Queer Studies o Gay Studies, sia all'identità omosessuale femminile, dalla quale si è sviluppato il campo dei Lesbian Studies» ${ }^{42}$, con una equivalenza livellante fra queer studies e gay studies.

Resta però un rilievo da fare: questi pochi accenni sono comunque segnale di una frattura nel muro di gomma delle metodologie ufficiali, di una traversa consapevolezza che comincia a incrinare i capitoli stretti della maggioranza dei manuali. Anche perché non può non essere registrata una inversione. Di tendenza, questa volta.

\section{Tendencies $^{43}$ : vista sulle prospettive}

Mentre fuori dai confini nazionali ci si interroga sulla posterità queer, questo avviene senza che il termine sia stato 'consumato', in Italia, all'interno di acquisizioni e applicazioni accademiche riconoscibili. Soltanto negli ultimi anni si è assistito ad una sorta di fioritura ${ }^{44}$, un acceso interesse grazie al lavoro (e in alcuni casi alla pervicacia) di una nuova generazione di studiosi, una sorta di queer generation ${ }^{45}$ che, assieme a studiosi e militanti noti, in virtù delle proprie auto-narrazioni critiche sta cominciando a interrogare $\mathrm{i}$ testi da un punto di vista epistemologicamente dissidente. Questo movimento ha fatto sì che, in pochi anni, si sia dato

40. Marco Pustianaz, Teoria gay e lesbica, in Teoria della letteratura. Prospettive dagli Stati Uniti, cit.

4I. Massimo Fusillo, Estetica della letteratura, Bologna, Il Mulino, 2009, pp. I57-I59.

42. Gino Tellini, Metodi e protagonisti della critica letteraria. Con antologia di testi e prove di lettura, Firenze, Le Monnier, 20Io, p. 26I.

43. "And everywhere at the march, on women and on men, there were T-shirts that said simply: QUEER. It was a QUEER time.» (Eve Kosofsky Sedgwick, Tendencies, London, Routledge, I994, p. XI.)

44. Che ha visto in Italia, per esempio, la Società delle Storiche e le discipline sociali e psicologiche in prima linea.

45. Di questo ho parlato in Eleonora Pinzuti, I Queer Studies, in Introduzione della letteratura, a cura di Franco Brioschi, Costanzo di Girolamo, Massimo Fusillo, Roma, Carocci, in corso di stampa. 
vita a convegni, giornate di studio, seminari nazionali e non, che hanno avuto come argomento i queer studies. Penso, senza pretesa di esaustività e solo a titolo di esempio, all'incontro che si tenne all'Istituto Italiano di Cultura di Budapest nel 2009, che ospitò un intervento sui queer studies, ma soprattuto al convegno internazionale Queer Crossing che si è tenuto a Palermo il I8-I9 giugno 20IO; a Lo Spazio della differenza svoltosi all'Università di Milano Bicocca nell'ottobre del 20Io; al convegno annuale della Compalit (Associazione per gli Studi di Teoria e Storia Comparata della Letteratura) svoltosi all'Università di Messina dal I8 al 20 novembre 2010 che ha dedicato una sessione (Performance dell'Identità) anche ai queer studies; al convegno dell'Université Stendhal-Grenoble 3 On ne naît pas... on le devient, di cui questo volume fa fede, che sempre nel novembre del 20 o affrontava i temi del gender e del queer proprio nell'ambito degli studi di Italianistica. Nel 20II a Firenze, presso il Dipartimento di Studi Storici e Geografici, si è tenuta il 25 febbraio la prima giornata interamente dedicata a Storia dei lesbismi e studi lgbtq in Italia (dove "q" sta per queer, oramai inserito nell'acronimo), mentre in marzo (il I7 e il I8) sempre a Firenze si è svolto il primo convegno internazionale dedicato a L'arte del desiderio. Omosessualità, letteratura, differenza. Nell'ottobre del $20 I$ si è tenuto un workshop a Bristol dal titolo Quando eravamo froci. Queering the Peninsula al quale hanno partecipato studiosi di Italianistica assai impegnati su questo versante come Derek Duncan ${ }^{46}$ e Charlotte Ross ${ }^{47}$, mentre nel novembre sempre del 201 a a Padova si è svolto un convegno dal titolo Inquietudini queer. Desiderio, performance, scrittura (e questo elenco non è certo esaustivo né rende merito a tutt* i protagonisti di questa fioritura).

Sul versante delle pubblicazioni, anche qui per ricordare solo alcuni degli studi ora a disposizione, hanno visto la luce la serie Omosapiens ${ }^{48}$ pubblicata da Carocci, il volume Oltre l'identità sessuale. Teorie queer e corpi transgender di Flavia Monceri ${ }^{49}$, quello collettivo Queer in Italia. Differenze in movimento ${ }^{50}$ a cura di Marco Pustianaz. Mentre scrivo sono

46. Di Derek Duncan va ricordato il suo Reading \& Writing Italian Homosexualities. A Case of Possible Difference, Aldershot, Burlington (VT), Ashgate, 2006.

47. Importante, fra gli altri contributi, per gli studi di Italianistica, il numero speciale di «Italian Studies» che Charlotte Ross ha curato con Susanna Scarparo, Gender and Sexuality in Contemporary Italian Culture: Representations and Critical Debates, 65, 2, July 2010.

48. Si tratta di tre volumi: OmoSapiens, Studi e ricerche sugli orientamenti sessuali, a cura di Domenico Rizzo, Roma, Carocci, 2006; OmoSapiens 2. Spazi e identità queer, a cura di Silvia Antosa, Roma, Carocci, 2007; OmoSapiens 3. Per una sociologia dell'omosessualità, a cura di Luca Trappolin, Roma, Carocci, 2008.

49. Flavia Monceri, Oltre l'identità sessuale. Teorie queer e corpi transgender, Pisa, ETS, 2010.

50. Queer in Italia. Differenze in movimento, a cura di Marco Pustianaz, cit. 
inoltre in preparazione gli atti dei convegni sopra ricordati, e dunque nel corso del 20I2-20I3 molti altri saranno gli studi e gli stimoli con i quali confrontarsi.

Inoltre, sul versante della traduzione, abbiamo finalmente nuovi strumenti. È uscita infatti nel 2010 una antologia dei più importanti scritti di Judith/Jack Halberstam, Maschilità senza uomini, a cura di Federica Frabetti $^{\text {sI }}$, e abbiamo a disposizione la traduzione di un testo cardine dei queer studies come Epistemology of the Closet di Eve Kosofsky Sedgwick, dal titolo Stanze private. Epistemologia e politica della sessualità ${ }^{52}$, che darà un contributo essenziale alla discussione metodologica.

Tutti questi strumenti, questi incroci e questi confronti vivono nell'intersecazione stessa della epistemologia, delle discipline e dei testi, mettendo in discussione il vecchio monologismo e il finalismo euristico/ideologico che lo prevedeva come pre-condizione interpretativa. Del resto i queer studies, nati in ambito americano da un intervento oramai leggendario di Teresa de Lauretis ${ }^{53}$, sono una modalità di conoscenza non saturabile ed eccedente, che si muove nel continuo spostamento delle dittologie e gioca con la permeabilità del concetto stesso di interpretazione, cassandone il «residuo teologale» ${ }^{54}$. Il testo diventa così il campo principe per valutare la tenuta delle soggettività, delle identità, delle politiche e delle storie e viene attivato nella sua materialità culturale (nelle sue interrogazioni storiche, temporali) così come nelle sue materializzazioni. La comparsa oggi di un soggett* asteriscato implicitamente posto oltre il dualismo e che si relaziona in evidente modalità antiautoritaria (in linea prossemica col testo) produce studi volti a decostruire ogni posizione forzosamente inserita all'interno di un contesto pre-ordinato, comprese le declinazioni ermeneutiche proprie del soggetto dominante e le sue funzioni retoriche. In questo senso i queer studies, comprendendo la soggettivazione gay e lesbica come identità in movimento, sottraggono a qualsiasi violenza epistemologica non tanto l'oramai liquida categoria dell'identità, quanto la 'fissità' stessa dei 'saperi monumentali', evidenziando la loro consunzione e la loro natura im-permanente. Infatti il testo non rappresenta più, per questa nuova generazione, solo e soltanto l'idolo o il feticcio (quando non manzonianamente la vittima di tante violenze ermeneutiche) quanto una

5I. Judith Halberstam, Maschilità senza uomini. Scritti scelti, a cura di Federica Frabetti, Pisa, ETS, 2010.

52. Eve Kosofsky Sedgwick, Stanze private. Epistemologia e politica della sessualità, a cura di Federico Zappino, introduzione di Silvia Antosa, Roma, Carocci, 201 .

53. Teresa de Lauretis, Queer Theory: Lesbian and Gay Sexualities. An Introduction, in «Differences. A Journal of Feminist Cultural Studies», 3, 2, I99I, pp. III-XVIII.

54. Antonio Gramsci, Lettere dal carcere, Torino, Einaudi, I97I (edizione originale 1947), p. 226. 
piattaforma di sollecitazioni epistemiche da rinegoziare volta per volta. Del resto, la letteratura o è processo di comparazione (simbolico, storico, politico e testuale) o non è, fuori e dentro l'istituzione accademica. Bisogna rileggere l'intera teoria letteraria anche come soggettivazione delle inquietudini storiche e politiche, abbandonando quella inafferrabile "teoria oggettiva» a cui molti tradizionalisti restano legati. E soprattutto bisogna inserire all'interno della lettura una sorta di teoria dell'inquietudine, di soggett*, di nazioni o di interi sistemi culturali, perché pare difficile non ammettere che «la più alta come la più bassa forma di critica sono una specie di autobiografia»ss. 